PROCEEDINGS OF THE

AMERICAN MATHEMATICAL SOCIETY

Volume 130, Number 7 , Pages $2007-2013$

S 0002-9939(01)06254-2

Article electronically published on December 27, 2001

\title{
ON SUMS OF DARBOUX AND NOWHERE CONSTANT CONTINUOUS FUNCTIONS
}

\author{
KRZYSZTOF CIESIELSKI AND JANUSZ PAWLIKOWSKI
}

(Communicated by Alan Dow)

\begin{abstract}
We show that the property
(P) for every Darboux function $g: \mathbb{R} \rightarrow \mathbb{R}$ there exists a continuous nowhere constant function $f: \mathbb{R} \rightarrow \mathbb{R}$ such that $f+g$ is Darboux follows from the following two propositions:

(A) for every subset $S$ of $\mathbb{R}$ of cardinality c there exists a uniformly continuous function $f: \mathbb{R} \rightarrow[0,1]$ such that $f[S]=[0,1]$,

(B) for an arbitrary function $h: \mathbb{R} \rightarrow \mathbb{R}$ whose image $h[\mathbb{R}]$ contains a nontrivial interval there exists an $A \subset \mathbb{R}$ of cardinality $\mathfrak{c}$ such that the restriction $h\lceil A$ of $h$ to $A$ is uniformly continuous,

which hold in the iterated perfect set model.
\end{abstract}

Our set theoretic terminology is standard and follows that of [3]. In particular, functions are identified with their graphs and $|X|$ stands for the cardinality of a set $X$. The symbol $\mathfrak{c}$ stands for $|\mathbb{R}|$ and $\mathcal{C}(\mathbb{R})$ denotes the family of all continuous functions from $\mathbb{R}$ into $\mathbb{R}$. A function $f: \mathbb{R} \rightarrow \mathbb{R}$ is Darboux if a conclusion of the intermediate value theorem holds for $f$ or, equivalently, when $f$ maps every interval onto an interval; $f$ is a Sierpiński-Zygmund function if its restriction $f \uparrow Y$ is discontinuous for every subset $Y$ of $\mathbb{R}$ of cardinality $\mathfrak{c} ; f$ is nowhere constant if it is not constant on any non-trivial interval.

The class of Darboux functions has been studied for a long time as one of possible generalizations of the class of continuous functions. However, it has some peculiar properties. For example, it is not closed under addition. In fact, in 1927 Lindenbaum [8] noticed that every function $f: \mathbb{R} \rightarrow \mathbb{R}$ can be written as a sum of two Darboux functions (see also [9]) while Erdős [5] showed that if additionally $f$ is measurable, then both of the summands can be chosen to be measurable. In 1990 Kirchheim and Natkaniec showed that there are some truly bad Darboux functions by proving the following.

Proposition 1 (Kirchheim, Natkaniec [6]). If the union of less than $\mathfrak{c}$ many meager subsets of $\mathbb{R}$ is meager (thus under $\mathrm{CH}$ or $\mathrm{MA}$ ), then there exists a Darboux function $g: \mathbb{R} \rightarrow \mathbb{R}$ such that $f+g$ is not Darboux for every continuous nowhere constant function $f: \mathbb{R} \rightarrow \mathbb{R}$.

Received by the editors November 13, 2000 and, in revised form, January 24, 2001.

1991 Mathematics Subject Classification. Primary 26A15; Secondary 03E35.

Key words and phrases. Darboux, nowhere constant, images of continuous functions.

The work of the second author was partially supported by KBN Grant 2 P03A 03114.

(C)2001 American Mathematical Society 
The problem of whether the additional set-theoretic assumptions are necessary in this theorem was investigated in 1992 by Komjáth [7] and was settled in 1995 by Steprāns.

Proposition 2 (Steprāns [11]). It is consistent with ZFC that

(P) for every Darboux function $g: \mathbb{R} \rightarrow \mathbb{R}$ there exists a continuous nowhere constant function $f: \mathbb{R} \rightarrow \mathbb{R}$ such that $f+g$ is Darboux.

In his paper Steprāns showed that $(\mathrm{P})$ holds in the iterated perfect set model. His direct forcing argument is quite complicated and difficult to follow. We will show that $(\mathrm{P})$ follows from the principles $(\mathrm{A})$ and $(\mathrm{B})$ defined in the abstract. Since the proofs that $(\mathrm{A})$ and $(\mathrm{B})$ hold in the the iterated perfect set model are much simpler than the argument given in [11], our argument for $(\mathrm{P})$ is essentially more accessible.

The proof that (A) holds in the iterated perfect set model is due to Miller [10]. The proof that (B) holds in this model is due to Balcerzak, Ciesielski, and Natkaniec 1] and consists of a page-long argument based on the results from [10. In addition, properties (A) and (B) follow quite easily from the Covering Property Axiom (CPA) as shown by the authors in [4. For more on the Darboux property see also the survey article [2].

Note also that condition (B) is equivalent to its following seemingly stronger version:

(B*) for an arbitrary $S \subset \mathbb{R}$ and a function $h: S \rightarrow \mathbb{R}$ whose image $h[S]$ contains a non-trivial interval there exists an $A \subset S$ of cardinality c such that the restriction $h \uparrow A$ of $h$ to $A$ is uniformly continuous.

Clearly ( $\mathrm{B}^{*}$ ) implies (B). To see the other implication let $h$ be as in (B*) and $f: \mathbb{R} \rightarrow \mathbb{R}$ be Sierpiński-Zygmund. Put $h^{*}=f \uparrow(\mathbb{R} \backslash S) \cup h$ and apply (B) to find $A^{*} \subset \mathbb{R}$ of cardinality c such that $h^{*}\left\lceil A^{*}\right.$ is uniformly continuous. Then $A=A^{*} \cap S$ is as in $\left(\mathrm{B}^{*}\right)$.

Our main theorem is the following.

Theorem 3. Assume that (A) and (B) hold. Then for every Darboux function $d: \mathbb{R} \rightarrow \mathbb{R}$ there exist a complete metric $\rho$ on $\mathcal{C}(\mathbb{R})$ and a dense $G_{\delta}$ subset $G$ of $\langle\mathcal{C}(\mathbb{R}), \rho\rangle$ of nowhere constant functions such that $d+g$ is Darboux for every $g \in G$.

In particular $(\mathrm{A}) \mathcal{E}(\mathrm{B})$ implies $(\mathrm{P})$.

Our proof will be based on the following two lemmas.

Lemma 4. Let $d: \mathbb{R} \rightarrow \mathbb{R}$ be Darboux and $D \subset \mathbb{R}$ be such that $d \uparrow D$ is dense in d. If $g: \mathbb{R} \rightarrow \mathbb{R}$ is continuous and such that

$$
[(d+g)(\alpha),(d+g)(\beta)] \subset(d+g)[[\alpha, \beta]] \text { for all } \alpha, \beta \in D,
$$

then $d+g$ is Darboux.

Proof. An easy proof can be found in [11, lemma 4.1].

Lemma 5. Assume that (A) holds and for every $n<\omega$ let $A_{n} \in[\mathbb{R}]^{\mathfrak{c}}$. Then for every $n<\omega$ there exists a $B_{n} \in\left[A_{n}\right]^{\mathfrak{c}}$ such that the closures of $B_{n}$ 's are pairwise disjoint.

Proof. First note that (A) implies

$$
\forall\left\langle C_{n} \in[\mathbb{R}]^{\mathfrak{c}}: n<\omega\right\rangle \exists C \in\left[C_{0}\right]^{\mathfrak{c}} \forall n<\omega\left|C_{n} \backslash \operatorname{cl}(C)\right|=\mathfrak{c} .
$$


Indeed, by condition (A), we can find a continuous function $f: \mathbb{R} \rightarrow[0,1]$ such that $\left|C_{0} \cap f^{-1}(c)\right|=\mathfrak{c}$ for every $c \in[0,1]$. (Just take a composition of a function from (A) with the Peano curve followed by a projection.) Identify $2^{\omega}$ with a subset of $[0,1]$ and by induction on $n<\omega$ choose an increasing sequence $s_{n} \in 2^{n+1}$ such that $\left|C_{n} \backslash f^{-1}\left(\left\{c \in 2^{\omega}: s_{n} \subset c\right\}\right)\right|=\mathfrak{c}$. Put $s=\bigcup_{n<\omega} s_{n}$. Then $C=C_{0} \cap f^{-1}(s)$ satisfies (2).

Next note that there exists a sequence $\left\langle D_{n}: n<\omega\right\rangle$ of closed subsets of $\mathbb{R}$ such that $\left|A_{n} \cap\left(D_{n} \backslash \bigcup_{i<n} D_{i}\right)\right|=\mathfrak{c}$. This sequence is constructed by using induction on $n<\omega$ with each set $D_{n}$ chosen by applying (2) to the sequence $\left\langle A_{k} \backslash \bigcup_{i<n} D_{i}: n \leq k<\omega\right\rangle$.

Finally, since each set $D_{n} \backslash \bigcup_{i<n} D_{i}$ is an $F_{\sigma}$-set, we can find closed subsets of them, $E_{n}$, with $B_{n}=E_{n} \cap A_{n}$ having cardinality $\mathfrak{c}$. It is easy to see that the sets $B_{n}$ are as required.

Remark 6. Note that Lemma 5 cannot be proved in ZFC. Indeed, if we assume that there exists a $\mathfrak{c}$-Luzin set $\mathbb{1}^{1}$ and $\left\langle A_{n} \in[\mathbb{R}]^{\mathfrak{c}}: n\langle\omega\rangle\right.$ is a sequence of $\mathfrak{c}$-Luzin sets such that every non-empty open interval contains one of $A_{n}$ 's, then for such a sequence there are no $B_{n}$ 's as in the lemma.

Lemma 7. Assume that (A) and (B) hold and $d: \mathbb{R} \rightarrow \mathbb{R}$ is a Darboux function. If $D$ is a countable subset of $\mathbb{R}$ such that

$(*)$ for every $\alpha<\beta$ from $D$ for which $d(\alpha)=d(\beta)$ there exist $p, q \in \mathbb{R}$ such that $\alpha<p<q<\beta$ and $d[(p, q)]=\{f(\alpha)\}$,

then there exists a countable family $\mathcal{A} \subset[\mathbb{R}]^{\mathfrak{c}}$ such that

(a) different elements of $\mathcal{A}$ have disjoint closures,

(b) $d \uparrow A$ is uniformly continuous for every $A \in \mathcal{A}$, and

(c) for every $\alpha, \beta \in D$ there exists an $A \in \mathcal{A}$ with the property that $d\lceil A \subset$ $[\alpha, \beta] \times[d(\alpha), d(\beta)]$.

Proof. First for every $\alpha<\beta$ from $D$ we choose $A_{\alpha}^{\beta} \in[\mathbb{R}]^{\mathfrak{c}}$ such that the family $\mathcal{A}_{0}=\left\{A_{\alpha}^{\beta}: \alpha, \beta \in D \& \alpha<\beta\right\}$ satisfies (b) and (c). For this fix $\alpha<\beta$ from $D$.

If $d(\alpha)=d(\beta)$, then it is enough to put $A_{\alpha}^{\beta}=(p, q)$, where $p$ and $q$ are from $(*)$. So, assume that $d(\alpha) \neq d(\beta)$. Then $I=[d(\alpha), d(\beta)]$ is a non-trivial interval, which is a subset of $d[[\alpha, \beta]]$, since $d$ is Darboux. Let $S=[\alpha, \beta] \cap d^{-1}(I)$ and notice that $h=d\left\lceil S\right.$ maps $S$ onto $I$. So, by $\left(\mathrm{B}^{*}\right)$, we can find an $A_{\alpha}^{\beta} \in[S]^{\mathrm{c}}$ such that $d \uparrow A_{\alpha}^{\beta}$ is uniformly continuous.

It is easy to see that $\mathcal{A}_{0}$ satisfies (b) and (c) so it is enough to decrease its elements to get condition (a), while assuring that they still have cardinality $\mathfrak{c}$. This can be done by applying Lemma 5 .

Proof of Theorem 3 . Take a Darboux function $d: \mathbb{R} \rightarrow \mathbb{R}$ and choose a countable dense set $D \subset \mathbb{R}$ satisfying condition (*) from Lemma 7 such that $d \uparrow D$ is dense in $d$. Let $\mathcal{A} \subset[\mathbb{R}]^{\mathfrak{c}}$ be the family from Lemma 7 and let $\left\{A_{n}: n<\omega\right\}$ be an enumeration of $\mathcal{A} \cup\{\{d\}: d \in D \backslash \cup \mathcal{A}\}$. Let $\rho_{0}$ be the uniform convergence metric on $\mathcal{C}(\mathbb{R})$, that is,

$$
\rho_{0}(f, g)=\min \{1, \sup \{|f(x)-g(x)|: x \in \mathbb{R}\}\}
$$

\footnotetext{
${ }^{1}$ A set $L \subset \mathbb{R}$ is a $\mathfrak{c}$-Luzin set if $|L|=\mathfrak{c}$ but $|L \cap N|<\mathfrak{c}$ for every nowhere dense subset $N$ of $\mathbb{R}$. It is well known (see, e.g., [10, sec. 2]) and easy to see that no c-Luzin set can be mapped continuously onto $[0,1]$. Thus (A) implies that there is no $\mathfrak{c}$-Luzin set.
} 
and, for $f, g \in \mathcal{C}(\mathbb{R})$, let

$$
\rho_{1}(f, g)=2^{-\min \left\{n<\omega: f \nmid A_{n} \neq g \mid A_{n}\right\}} .
$$

(If $\left\{n<\omega: f\left|A_{n} \neq g\right| A_{n}\right\}=\emptyset$ we assume that $\rho_{1}(f, g)=0$.) Then $\rho_{1}$ is a pseudometric on $\mathcal{C}(\mathbb{R})$. Consider $\mathcal{C}(\mathbb{R})$ with the following metric $\rho$ :

$$
\rho(f, g)=\max \left\{\rho_{0}(f, g), \rho_{1}(f, g)\right\}
$$

and notice that $\langle\mathcal{C}(\mathbb{R}), \rho\rangle$ forms a complete metric space. We will prove that if $G$ is the set of all nowhere constant continuous functions $g: \mathbb{R} \rightarrow \mathbb{R}$ for which $d+g$ satisfies (1) of Lemma 4 then $G$ contains a dense $\mathrm{G}_{\delta}$ subset of $\langle\mathcal{C}(\mathbb{R}), \rho\rangle$. For this we will show that for every $\alpha<\beta$ from $D$ the following two types of sets contain dense open subsets of $\langle\mathcal{C}(\mathbb{R}), \rho\rangle$ :

$$
H_{\alpha}^{\beta}=\{g \in \mathcal{C}(\mathbb{R}): g \text { is not constant on }(\alpha, \beta)\}
$$

and

$$
G_{\alpha}^{\beta}=\{g \in \mathcal{C}(\mathbb{R}):[(d+g)(\alpha),(d+g)(\beta)] \subset(d+g)[[\alpha, \beta]]\}
$$

Then

$$
\bigcap\left\{H_{\alpha}^{\beta} \cap G_{\alpha}^{\beta}: \alpha, \beta \in D \& \alpha<\beta\right\} \subset G
$$

will contain a dense $\mathrm{G}_{\delta}$ subset of $\langle\mathcal{C}(\mathbb{R}), \rho\rangle$.

To see that $H_{\alpha}^{\beta}$ contains a dense open subset first note that, by (a) and (c) of Lemma 7 elements of $\mathcal{A}$ are nowhere dense. Next, take an $f \in \mathcal{C}(\mathbb{R})$, and fix an $\varepsilon>0$. Let $B(f, \varepsilon)$ be the open $\rho$-ball centered at $f$ and of radius $\varepsilon$. We will find $g \in B(f, \varepsilon)$ and $\delta>0$ such that $B(g, \delta) \subset B(f, \varepsilon) \cap H_{\alpha}^{\beta}$. So take an $n<\omega$ such that $2^{-n}<\varepsilon / 4$, choose a non-empty open interval $J \subset(\alpha, \beta) \backslash \bigcup_{i=1}^{n} A_{i}$, and pick different $x, y \in J$. It is easy to find $g \in \mathcal{C}(\mathbb{R})$ such that $g(x) \neq g(y), g\lceil(\mathbb{R} \backslash J)=f \uparrow(\mathbb{R} \backslash J)$, and $\rho_{0}(f, g)<2^{-n}$. Then, by the choice of $J$ we also have $\rho_{1}(f, g)<2^{-n}$, so $g \in B(f, \varepsilon / 4)$. Now, if $\delta=\min \{|g(x)-g(y)| / 4, \varepsilon / 4\}$, then $B(g, \delta) \subset B(f, \varepsilon) \cap H_{\alpha}^{\beta}$.

To see that each $G_{\alpha}^{\beta}$ contains a dense open subset take $f \in \mathcal{C}(\mathbb{R})$ and $\varepsilon>0$. As previously we will find $g \in B(f, \varepsilon)$ and $\delta>0$ such that $B(g, \delta) \subset B(f, \varepsilon) \cap G_{\alpha}^{\beta}$. Find $\alpha=x_{0}<x_{1}<\cdots<x_{m}=\beta, x_{i} \in D$, such that the variation of $f$ on each interval $\left[x_{i}, x_{i+1}\right]$ is less than $\varepsilon / 8$. Also, since $d \uparrow\left[x_{i}, x_{i+1}\right]$ is Darboux, we can partition each $\left[x_{i}, x_{i+1}\right]$ even further to also get that $\left|d\left(x_{i}\right)-d\left(x_{i+1}\right)\right|<\varepsilon / 8$ for all $i<m$.

Pick an $n<\omega$ such that $2^{-n}<\varepsilon / 8$ and $\left\{x_{i}: i \leq m\right\} \subset \bigcup_{i=1}^{n} A_{i}$. For every $i<m$ choose an index $k_{i}>n$ such that $d\left\lceil A_{k_{i}} \subset\left(x_{i}, x_{i+1}\right) \times\left[d\left(x_{i}\right), d\left(x_{i+1}\right)\right]\right.$. By (A) for every $i<m$ we can also pick a uniformly continuous function $h_{i}$ from $A_{k_{i}}$ onto $\left[(d+f)\left(x_{i}\right),(d+f)\left(x_{i+1}\right)\right]$. For each $i<m$ define $h\left\lceil A_{k_{i}}=\left(h_{i}-d-f\right) \uparrow A_{k_{i}}\right.$ and note that

$$
h\left[A_{k_{i}}\right] \subset h_{i}\left[A_{k_{i}}\right]-d\left[A_{k_{i}}\right]-f\left[A_{k_{i}}\right] \subset[-\varepsilon / 2, \varepsilon / 2]
$$

since $\left[(d+f)\left(x_{i}\right),(d+f)\left(x_{i+1}\right)\right] \subset\left[d\left(x_{i}\right)+f\left(x_{i}\right)-\varepsilon / 4, d\left(x_{i}\right)+f\left(x_{i}\right)+\varepsilon / 4\right], d\left[A_{k_{i}}\right] \subset$ $\left[d\left(x_{i}\right), d\left(x_{i+1}\right)\right] \subset\left[d\left(x_{i}\right)-\varepsilon / 8, d\left(x_{i}\right)+\varepsilon / 8\right]$, and $f\left[A_{k_{i}}\right] \subset\left(f\left(x_{i}\right)-\varepsilon / 8, f\left(x_{i}\right)+\varepsilon / 8\right)$. Also define $h$ as 0 on $\bigcup_{i=1}^{n} A_{i}$ and extend it to a uniformly continuous function from $\mathbb{R}$ into $[-\varepsilon / 2, \varepsilon / 2]$. Put $g=f+h$ and note that $\rho(f, g) \leq \varepsilon / 2$. Also let $k=\max \left\{n, k_{0}, \ldots, k_{m-1}\right\}$ and $\delta \in\left(0,2^{-k}\right)$. We claim that $B(g, \delta) \subset B(f, \varepsilon) \cap G_{\alpha}^{\beta}$.

Indeed, it is easy to see that $B(g, \delta) \subset B(f, \varepsilon)$. To see that $B(g, \delta) \subset G_{\alpha}^{\beta}$ take a $g_{0} \in B(g, \delta)$. By the choice of $\delta, h$, and $g$ for every $i \leq m$ we have

$$
\left(d+g_{0}\right)\left(x_{i}\right)=(d+g)\left(x_{i}\right)=(d+f+h)\left(x_{i}\right)=(d+f)\left(x_{i}\right)
$$


and, for $A=\bigcup_{j<m} A_{k_{j}}$,

$$
g_{0}\left\lceil A=g \uparrow A=(f+h) \uparrow A=\left(f+\left(h_{i}-d-f\right)\right) \uparrow A=\left(h_{i}-d\right) \uparrow A .\right.
$$

So,

$$
\begin{aligned}
{\left[\left(d+g_{0}\right)(\alpha),\left(d+g_{0}\right)(\beta)\right] } & =\left[(d+f)\left(x_{0}\right),(d+f)\left(x_{m}\right)\right] \\
& \subset \bigcup_{j<m}\left[(d+f)\left(x_{j}\right),(d+f)\left(x_{j+1}\right)\right] \\
& =\bigcup_{j<m} h_{j}\left[A_{k_{j}}\right] \\
& =\bigcup_{j<m}\left(d+\left(h_{j}-d\right)\right)\left[A_{k_{j}}\right] \\
& =\bigcup_{j<m}\left(d+g_{0}\right)\left[A_{k_{j}}\right] \\
& \subset\left(d+g_{0}\right)[[\alpha, \beta]]
\end{aligned}
$$

proving that $g_{0} \in G_{\alpha}^{\beta}$. This finishes the proof of the theorem.

We say that a function $f: \mathbb{R} \rightarrow \mathbb{R}$ is a $D^{1}$ function if it is differentiable with its derivative $f^{\prime}(x)$ finite at every $x \in \mathbb{R} ; f$ is in the class " $D^{1}$ " provided its derivative $f^{\prime}(x)$ exists at every point, but it can have an infinite value. We say that $f$ belongs to $\mathcal{C}^{1}$ (to " $\mathrm{C}^{1}$ ") if it belongs to $D^{1}$ (to " $D^{1}$ ", respectively) and its derivative $f^{\prime}$ is continuous.

Let us also notice that in $(\mathrm{P})$ we cannot require that function $g$ is " $\mathrm{C}^{1}$ ". This follows from the following fact which, for the functions from the class $\mathcal{C}^{1}$, was first noticed by Steprāns [11, p. 118]. Since Steprāns leaves his statement without any comments concerning its proof, we include here a missing argument.

Proposition 8. There exists, in ZFC, a Darboux function $d: \mathbb{R} \rightarrow \mathbb{R}$ such that $d+f$ is not Darboux for every non-constant " $\mathcal{C}$ " function $f$.

Proof. Let $\mathbb{R}=\left\{x_{\xi}: \xi<\mathfrak{c}\right\}$ and let $\left\{f_{\xi}: \xi<\mathfrak{c}\right\}$ be an enumeration of all nonconstant " $\mathcal{C}$ " functions. Notice that for every $\xi<\mathfrak{c}$ there exists a non-empty open interval $I_{\xi}$ such that $f_{\xi}\left\lceil I_{\xi}\right.$ is strictly monotone. (Just take an $x \in \mathbb{R}$ such that $f_{\xi}^{\prime}(x) \neq 0$, which exists since $f_{\xi}$ is non-constant. Then $I_{\xi}$ is chosen as a neighborhood of $x$ on which $f_{\xi}^{\prime}$ is non-zero.)

The function $d$ that we construct will be strongly Darboux in a sense that $d^{-1}(r)$ is dense in $\mathbb{R}$ for every $r \in \mathbb{R}$. For such $d$ in order to show that $d+f_{\xi}$ is not Darboux it is enough to show that $\left(d+f_{\xi}\right)^{-1}\left(y_{\xi}\right)$ is not dense in $\mathbb{R}$ for some $y_{\xi} \in \mathbb{R}$. (See, e.g., [3, prop. 7.2.4].)

By induction we construct a sequence $\left\{\left\langle Q_{\xi}, d_{\xi}, y_{\xi}\right\rangle: \xi<\mathfrak{c}\right\}$ such that for every $\xi<\mathfrak{c}$ we have:

(i) Sets $\left\{Q_{\eta}: \eta \leq \xi\right\}$ are countable and pairwise disjoint, and $x_{\xi} \in \bigcup_{\eta \leq \xi} Q_{\eta}$.

(ii) $d_{\xi}: Q_{\xi} \rightarrow \mathbb{R}$ and $d_{\xi}^{-1}\left(x_{\xi}\right)$ is dense in $\mathbb{R}$.

(iii) $y_{\zeta} \notin\left(d_{\eta}+f_{\zeta}\right)\left[Q_{\eta} \cap I_{\zeta}\right]$ for every $\zeta, \eta \leq \xi$.

Notice that if we have such a sequence, then, by (i), $\mathbb{R}=\bigcup_{\xi<c} Q_{\xi}$ so $d=$ $\bigcup_{\xi<\mu} d_{\xi}: \mathbb{R} \rightarrow \mathbb{R}$. It is strongly Darboux by condition (ii), while, by (iii), for every 
$\zeta<\mathfrak{c}$ the set $\left(d+f_{\zeta}\right)^{-1}\left(z_{\zeta}\right)$ is not dense in $\mathbb{R}$, since it misses $I_{\zeta}$. Thus, $d$ is as desired.

To make an inductive $\xi$-th step first choose a countable dense subset $Q_{\xi}^{0}$ of $\mathbb{R}$ disjoint with

$$
\bigcup_{\eta<\xi} Q_{\eta} \cup \bigcup_{\zeta<\xi}\left(I_{\zeta} \cap f_{\zeta}^{-1}\left(y_{\zeta}-x_{\xi}\right)\right)
$$

This can be done since, by the choice of of the intervals $I_{\zeta}$ 's, each of the sets $I_{\zeta} \cap f_{\zeta}^{-1}\left(y_{\zeta}-x_{\xi}\right)$ has at most one element.

Define $d_{\xi}\left\lceil Q_{\xi}^{0}\right.$ as constantly equal to $x_{\xi}$. This guarantees (ii), while condition (iii) is satisfied for the part defined so far: for every $\zeta<\xi$ and $x \in I_{\zeta} \cap Q_{\xi}^{0}$ we have $d_{\xi}(x)+f_{\zeta}(x)=f_{\zeta}(x)+x_{\xi} \neq y_{\zeta}$, since otherwise we would have $x \in I_{\zeta} \cap f_{\zeta}^{-1}\left(y_{\zeta}-x_{\xi}\right)$ contradicting the choice of $Q_{\xi}^{0}$.

If $x_{\xi} \in \bigcup_{\eta<\xi} Q_{\eta}$ we define $Q_{\xi}=Q_{\xi}^{0}$. Otherwise we put $Q_{\xi}=Q_{\xi}^{0} \cup\left\{x_{\xi}\right\}$ and if $d_{\xi}\left(x_{\xi}\right)$ is not defined yet (i.e., if $\left.x_{\xi} \notin Q_{\xi}^{0}\right)$ we define $d_{\xi}\left(x_{\xi}\right)$ by choosing $d_{\xi}\left(x_{\xi}\right) \notin$ $\left\{y_{\zeta}-f_{\zeta}\left(x_{\xi}\right): \zeta<\xi\right\}$. This guarantees that (i) is satisfied, while (iii) is preserved.

Finally, we choose $y_{\xi}$ to have

$$
y_{\xi} \in \mathbb{R} \backslash \bigcup_{\eta \leq \xi}\left(d_{\eta}+f_{\xi}\right)\left[Q_{\eta}\right]
$$

This will guarantee that (iii) also holds for $\zeta=\xi$.

Let us also note that the following question, for the case of $D^{1}$ due to Steprāns [11, Question 5.1], remains open.

Problem 9. Does there exist, in ZFC, a Darboux function $d: \mathbb{R} \rightarrow \mathbb{R}$ such that $d+f$ is not Darboux for every nowhere constant " $D$ " " function $f$ ? What if we restrict the choice of $f$ to $D^{1}$ ?

\section{REFERENCES}

[1] M. Balcerzak, K. Ciesielski, T. Natkaniec, Sierpiński-Zygmund functions that are Darboux, almost continuous, or have a perfect road, Arch. Math. Logic 37 (1997), 29-35. (Preprint available in electronic form from Set Theoretic Analysis Web Page:

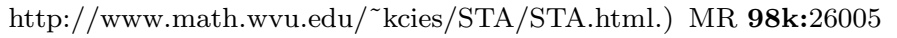

[2] K. Ciesielski, Set theoretic real analysis, J. Appl. Anal. 3(2) (1997), 143-190. (Preprint available in electronic form from Set Theoretic Analysis Web Page: http://www.math.wvu.edu/ ${ }^{\circ}$ kcies/STA/STA.html.) MR 99k:03038

[3] K. Ciesielski, Set Theory for the Working Mathematician, London Math. Soc. Stud. Texts 39, Cambridge Univ. Press, 1997. MR 99c:04001

[4] K. Ciesielski, J. Pawlikowski, Covering property axiom CPA, version of March 2001, work in progress. (Preprint available in electronic form from Set Theoretic Analysis Web Page: http://www.math.wvu.edu/ $\left.{ }^{\mathrm{k}} \mathrm{kies} / \mathrm{STA} / \mathrm{STA} . h t m l.\right)$

[5] P. Erdős, On two problems of S. Marcus concerning functions with the Darboux property, Rev. Roumaine Math. Pures Appl. 9 (1964), 803-804. MR 31:5944

[6] B. Kirchheim, T. Natkaniec, On universally bad Darboux functions, Real Anal. Exchange 16 (1990-91), 481-486. MR 92d:26011

[7] P. Komjáth, A note on Darboux functions, Real Anal. Exchange 18 (1992-93), 249-252. MR 94f:26001

[8] A. Lindenbaum, Sur quelques propriétés des fonctions de variable réelle, Ann. Soc. Math. Polon. 6 (1927), 129-130.

[9] S. Marcus, Asupra unei teoreme de A. Lindenbaum si demonstrate de W. Sierpinski, Acad. R. P. Romîne 19 (1960), 551-554. MR 24:A192 
[10] A. W. Miller, Mapping a set of reals onto the reals, J. Symbolic Logic 48 (1983), 575-584. MR 84k:03125

[11] J. Steprāns, Sums of Darboux and continuous functions, Fund. Math. 146 (1995), 107-120. MR 95m:26005

Department of Mathematics, West Virginia University, Morgantown, West Virginia 26506-6310

E-mail address: K_Cies@math.wvu.edu

$U R L:$ http://www.math. wvu.edu/ ${ }^{\sim}$ kcies

Department of Mathematics, University of Wroceaw, Pl. Grunwaldzki 2/4, 50-384 Wroceaw, Poland - And - Department of Mathematics, West Virginia University, MorGANTOWn, West Virginia 26506-6310

E-mail address: pawlikow@math.uni.wroc.pl 